\title{
El machismo en las familias de la comunidad de Numpatkaim, Imaza, Amazonas, 2019
}

\section{Machismo in the families of the community of Numpatkaim, Imaza, Amazon, 2019}

\author{
${ }^{1}$ Ander Leiva Chávez Anjis, ${ }^{2}$ Leo Kunchikui Akuts, ${ }^{3}$ José Darwin Farje Escobedo
}

\section{RESUMEN}

El presente trabajo de investigación tuvo como propósito, identificar actitudes machistas en las familias de la comunidad de Numpatkaim, distrito de Imaza, provincia de Bagua, región Amazonas, durante el año 2019; la investigación es de naturaleza no experimental, con diseño descriptivo simple. La muestra estuvo conformada por 100 pobladores (50 padres y 50 madres de familias); el instrumento utilizado fue una escala de actitudes frente al machismo, se establecieron tres niveles de actitudes machistas: bajo, medio y alto. Los resultados obtenidos muestran que el 68\% de los encuestados manifiestan un alto nivel de actitudes machistas en la dimensión dominio masculino, y un reducido 1\% se ubica en el nivel bajo. En la dimensión roles domésticos y sociales, el 51\% muestran un alto nivel de actitudes machistas y solamente el $5 \%$ está en el nivel bajo; en la dimensión superioridad masculina, el 53\% de los padres y madres de familia muestran un alto nivel de actitudes machistas y sólo el 3\% se ubica en el nivel bajo; y finalmente, en la dimensión roles sexuales, el $43 \%$ dice tener un alto nivel de actitudes machistas, frente a un 7\% que se ubica en el nivel bajo; demostrando que existe una fuerte dependencia de la mujer hacia el hombre, asociada a la cosmovisión awajún, que considera al hombre más fuerte y que encabeza la familia; en tanto que, la mujer cumple tareas domésticas y tiene muchas restricciones en el entorno familiar.

Palabras clave: Actitudes machistas en la comunidad awajún.

\begin{abstract}
The purpose of this research work was to identify sexist attitudes in the families of the Numpatkaim community, Imaza district, Bagua province, Amazonas region, during 2019; the research is non-experimental in nature, with a simple descriptive design. The sample consisted of 100 residents (50 fathers and 50 mothers of families); The instrument used was a scale of attitudes towards machismo, three levels of machista attitudes were established: low, medium and high. The results obtained show that $68 \%$ of those surveyed show a high level of sexist attitudes in the male domain dimension, and a reduced $1 \%$ are located in the low level. In the domestic and social roles dimension, $51 \%$ show a high level of sexist attitudes and only 5\% are at the low level; In the male superiority dimension, $53 \%$ of fathers and mothers show a high level of sexist attitudes and only $3 \%$ are located at the low level; and finally, in the sex roles dimension, $43 \%$ say they have a high level of sexist attitudes, compared to $7 \%$ who are at the low level; demonstrating that there is a strong dependence of women on men, associated with the awajún worldview, which considers the man to be stronger and who heads the family; whereas, the woman fulfills domestic tasks and has many restrictions in the family environment.
\end{abstract}

Keywords: Sexist attitudes in the Awajún community.

\footnotetext{
Bachiller en Educación egresado de la Facultad de Educación y Ciencias de la Comunicación de la Universidad Nacional Toribio Rodríguez de Mendoza de Amazonas.

${ }^{2}$ Bachiller en Educación egresado de la Facultad de Educación y Ciencias de la Comunicación de la Universidad Nacional Toribio Rodríguez de Mendoza de Amazonas.

${ }^{3}$ Licenciado en Educación, Docente Asociado, Facultad de Educación y Ciencias de la Comunicación de la Universidad Nacional Toribio Rodríguez de Mendoza de Amazonas.
} 


\section{INTRODUCCIÓN}

La violencia contra las mujeres a nivel global, es una manifestación de las relaciones de poder históricamente de desigualdad entre mujeres y hombres, al respecto la Organización Mundial de la Salud (1998) refiere que: La violencia contra la mujer a lo largo de su ciclo vital con la consecuencia, esencialmente de las pautas culturales, en particular de los efectos perjudiciales de algunas prácticas tradicionales o consuetudinarias y de todos los actos de extremismo relacionados con la raza, el sexo, el idioma o la religión, que perpetúan la condición inferior que se asigna a la mujer en la familia, el lugar de trabajo, la comunidad y la sociedad.

La Declaración de Beijing en la IV Conferencia Mundial sobre las mujeres - ONU, donde llegan entre otras a las siguientes conclusiones y acuerdos:

i) Nosotros, los Gobiernos nos comprometemos a promover los objetivos de igualdad, desarrollo y paz para todas las mujeres del mundo, en interés de la humanidad; ii) reconocer las voces de las mujeres del mundo entero y teniendo en cuenta la diversidad de las mujeres y sus papeles y circunstancias, rindiendo homenaje a las mujeres que han allanado el camino, e inspirados por la esperanza que reside en la juventud del mundo...; iii) Adoptar las medidas que sean necesarias para eliminar todas las formas de discriminación contra las mujeres y las niñas, y suprimir todos los obstáculos para la igualdad de género y el avance y empoderamiento de las mujeres.

El machismo, que es la exposición de la violencia del hombre contra la mujer, muestra casos alarmantes en nuestro país; en lo que va del año, según el Ministerio de la mujer y poblaciones vulnerables (2019), el Boletín del Programa Nacional contra la Violencia Familiar y Sexual, muestra que de abril a julio de 2019, han ocurrido 99 feminicidios, superando ampliamente al año 2018, en el mismo período, representando una variación del 17\% comparativamente al año pasado; y asimismo, en relación a años anteriores, se aprecia que se va incrementando conforme pasan los años; situación preocupante, a pesar que últimamente, han elevado las políticas de equidad de género y lucha contra la violencia de la mujer.

La etnia awajún, asentada en el departamento de Amazona, históricamente conocida como una raza guerrera que no se dejó conquistar por los incas, tampoco por los españoles; se caracteriza por el predominio del hombre hacia la mujer, al respecto Castilla (2015), expresa que las responsabilidades que cumplen cada uno de ellos, en la tradición indígena awajún, la mujer cosecha la chacra, extrae la yuca y los frutos del monte, remueve la tierra, va a la pesca, alimenta a sus hijos; es la piedra angular de la salud de la familia; el hombre, se dedica la mayor parte del tiempo a la caza (mitayar). Es bueno mencionar también que ocurren muchas muertes de mujeres awajun en manos de sus parejas, esposos, amantes, y que en su mayoría están en el anonimato. Esta realidad, es la que nos ha motivado realizar el trabajo de investigación que atañe propiamente a la cultura awajún; por lo que, nos formulamos el siguiente problema: ¿De qué manera se presentan las actitudes machistas en las familias de la comunidad de Numpatkaim?

Entre los antecedentes considerados, se menciona a Montserrat y Casas (2017), que en su estudio sobre "El impacto de la violencia machista y procesos de recuperación en la infancia y la adolescencia, en las madres y en las relaciones maternofiliales"; concluye que existe un índice bajo de ocupación laboral que podemos identificar entre las mujeres que viven violencia machista; situación que ocasiona que sus hijos se encuentren en un estado de mucha más vulnerabilidad en cuanto al acceso a las necesidades básicas.

Por su parte, Marchal, Brando, Hidalgo y Tomás (2018), en su investigación sobre "Diseño y validación de un instrumento para medir actitudes machistas, violencia y estereotipos en adolescentes", concluyen que: los niños presentan mayores creencias legitimadoras de la violencia, que las niñas, y que están atribuidas a factores socioculturales relacionadas a la socialización de género; mientras que, Pintado \& Meza (2015), en su tesis titulada "Mujer aguaruna y participación pública: una aproximación desde las creencias mitológicas y su influencia en la formación de roles e identidades, en las comunidades aguarunas, Nieva - Amazonas"; las conclusiones a las que arribaron, fueron: a los varones, de acuerdo al mito de Etsa, les corresponde realizar actividades vinculantes al espacio público y político, básicamente en lo que ellos denominan "el arte para la guerra". Esporádicamente complementan la subsistencia familiar basada en la caza y la pesca, contribuyendo de esta forma en lo que se denomina "el arte para la vida", desplegado con mayor ahínco por las mujeres.

En las bases teóricas, se iniciará definiendo al "machismo", como una ideología que defiende y justifica la superioridad y el dominio del hombre sobre la mujer; exalta las cualidades masculinas, como agresividad, independencia y dominancia, mientras estigmatiza las cualidades femeninas, como debilidad, dependencia y sumisión (De la Rubia y Ramos, 2016). Por su parte, Lugo (1985), lo define 
como "las conductas y actitudes que se asocian a la masculinidad, donde es el varón el que goza de la fuerza y el poder". Existen muchas posiciones para definir "el machismo", para Castañeda (2007), es una ideología que defiende y justifica la superioridad y el dominio del hombre sobre la mujer, exalta las cualidades masculinas, como agresividad, independencia y dominancia, mientras estigmatiza las cualidades femeninas, como debilidad, dependencia y sumisión. (p. 53). Por su parte, Kohan (1970), sostiene que el machismo se refiere al énfasis o exageración de las características masculinas, a la hombría y la creencia en la superioridad del hombre. Para Torres y Torres (1998), agrega que, en esta sociedad, el hombre poseía todos los derechos y todos los poderes y la mujer constituía una propiedad más; la primacía del hombre ha perdurado en todas las clases sociales con mayor o menor incidencia. La mujer es considerada como un menor de edad "como una criatura", poco responsable, que necesitaba protección.

El "machismo" está presente desde tiempos ancestrales en la mayoría de las sociedades, y que se asocia directamente a la cultura de los individuos, pero cabe precisar que la educación juega un rol importante en ese afán de equiparar los roles de varones y mujeres, que es lo que debe primar en una sociedad moderna. Dada la complejidad del tema, hay una variedad de estudiosos de todos los rangos que se ocupan del tema; por lo que, se hace mención a algunas bases teóricas como: Teoría Adleriana del complejo de inferioridad-superioridad Se asocia directamente a un principio dialéctico de la inferioridad de la mujer y la superioridad del varón; es él quien toma las riendas, dada su superioridad frente a ella, quien adopta un rol de respeto, sumisión y subordinación; esta relación interpersonal, despoja o quita a la mujer, de la oportunidad de disfrutar a plenitud de los placeres absolutos del erotismo; pero en aquellas sociedades letradas, es ella la que se revela ante el poder del hombre, avalado en el machismo; en ese sentido, en una lucha permanente y constante entre ambos géneros, Adler (1971). La psicología moderna, por su parte, está encontrando con meridiana claridad, que esa cualidad varonil de poder y ambición no son innatos ni inalterables, éstos; sin embargo, insertados por la sociedad tempranamente en el niño, crea las condiciones adecuadas para una atmósfera empapada de deseos de poder. Así mismo, considera el esfuerzo neurótico por una masculinidad completa y un sentimiento de hombría como el resultado de un sentimiento de inferioridad. Toda persona tiene sentimientos de inferioridad; el carácter defensivo del machismo es obvio al observar sus rasgos culturales. Asimismo, la teoría de Yuval Noah (como se citó en Querol, 2015), tuvo mucha fuerza en los dos últimos siglos; y su abordaje sobre la génesis del machismo, lo resume en tres principios: a) la primera, asiente que el hombre asume, debido a su gran fuerza, tareas y trabajos más duros, debido a su fortaleza; sin embargo, a la luz de la actualidad, tienen varios cuestionamientos; b) la segunda, hace referencia directa a la hormona masculina, particularmente la testosterona; que le atribuye fuerza y agresividad, en consecuencia, tiene cualidades para la guerra, protege a la familia y tiene acceso a espacios de poder en la sociedad; c) Por último, basado en los estudios de Darwin, el principio de la selección natural, donde la lucha por la hegemonía se da para garantizar la decendencia.

Según la teoría de las actitudes de Ubillus (2014), tiene que ver con la apreciación positiva o negativa de una persona hacia otra y viceversa, está asociada básicamente a la cultura, la ideología y las creencias de una persona que forma parte de un grupo social. las actitudes en realidad son aprendidas primero en el seno de la familia y dependiendo de su fortaleza, perduran en el tiempo o en todo caso, dan acceso a otros modelos adquiridos en contextos diferentes, si es que los aprendidos inicialmente son débiles y volátiles; estando en este caso, frente a personas o individuos dependientes o heterónomos. Las actitudes, en adelante pasan a formar el sello de la personalidad que va a caracterizar a cada persona como única.

El estudio ha tomado como referente las dimensiones desarrolladas por Bustamante (1999), relacionadas directamente a las actitudes machistas que son: i) Actitudes hacia el dominio masculino, asociadas a la potestad o mandato ejercido por el hombre hacia las mujeres, en cualquier contexto o escenario personal y social; ii) Actitudes relacionadas con los roles domésticos y sociales, que se conectan directamente con las interrelaciones entre los miembros de la familia, y que tienen que ver con los roles del hogar y su proyección al contexto comunal - social; iii) Actitudes frente a la superioridad masculina, se relaciona predominantemente a las esferas intelectual, afectivo y laboral; y, iv) Finalmente se consideran a las actitudes de los roles sociales, se relacionan con las actitudes que se van modelando en el seno familiar relacionados al ejercicio y control de la sexualidad.

El poblador awajún vive en el bosque, afincados en grupos de casas dispersas unas de otras, que en promedio albergaban a aproximadamente 300 vivientes. Según Seymour-Smith (1991), “es relevante para el tema de las relaciones de género, la unidad doméstica puede abarcar a más de una familia, 
quienes participan en una economía doméstica unitaria y están unidos por vínculos consanguíneos, que lo denominan patáag”. En el matrimonio awajún, tradicionalmente, el joven mitayero (cazador) sin conversar con la probable pareja, iba al monte a cazar y después de la caza, habiendo demostrado a la comunidad y a la mujer, ser un buen cazador; entonces estaba apto para juntarse con ella porque demostró valentía y fuerza, y eso le gustaba al padre y a toda la familia de la novia, porque supuestamente, el hombre no lo va hacer sufrir con hambre u otras necesidades porque su esposo siempre estará presto a asistir con alimentos. Asimismo, agrega Fuller (2013) "el matrimonio ideal es entre primos cruzados, pues así el yerno es al mismo tiempo un sobrino cruzado que trabajaría óptimamente para su suegro", además, el matrimonio, pues, implicaba que el esposo se traslade a casa de los suegros y que trabaje para ellos un tiempo como pago por la novia. Luego, la pareja debe construir una casa y formar una nueva unidad El trabajo de investigación fue desarrollado con el objetivo de identificar actitudes machistas en las familias de la comunidad de Numpatkaim, Imaza, Amazonas; la información obtenida servirá para conocer de manera sistemática el machismo presente en las familias que participaron en el estudio.

\section{MATERIALES Y MÉTODOS}

La presente investigación es de naturaleza no experimental, con diseño descriptivo simple (Valderrama, 2010), el esquema es:

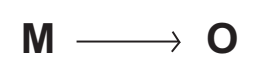

\section{Donde:}

M: Muestra de estudio

\section{O:Observación}

La población de estudio estuvo conformada por 100 pobladores varones y mujeres, con lengua materna awajún y que habitan en la comunidad de Numpatkaim, distrito de Imaza, provincia de Bagua. La muestra es igual a la población, conformada por 100 pobladores de la comunidad precitada.

En el desarrollo de la investigación se utilizó el método descriptivo "se orienta a construir toda una secuencia de hechos de manera prolija y sin alejarse de la realidad". El instrumento utilizado fue una encuesta comprendida por una escala de actitudes hacia el machismo, adaptado en bases a la propuesta de Bustamante (1999).
Tabla 1. Nivel de actitudes machistas en la dimensión dominio masculino.

\begin{tabular}{|c|c|c|}
\hline $\begin{array}{l}\text { Nivel de actitudes machistas: } \\
\text { dominio masculino }\end{array}$ & $\mathrm{f}$ & $\%$ \\
\hline Bajo & 1 & 1,0 \\
\hline Medio & 31 & 31,0 \\
\hline Alto & 68 & 68,0 \\
\hline Total & 100 & 100 \\
\hline
\end{tabular}

Tabla 2. Nivel de actitudes machistas en la dimensión roles domésticos.

\begin{tabular}{|c|c|c|}
\hline $\begin{array}{l}\text { Nivel de actitudes machistas: } \\
\text { dominio roles domésticos }\end{array}$ & $\mathrm{f}$ & $\%$ \\
\hline Bajo & 5 & 5,0 \\
\hline Medio & 44 & 44,0 \\
\hline Alto & 51 & 51,0 \\
\hline Total & 100 & 100 \\
\hline
\end{tabular}

Tabla 3. Nivel de actitudes machistas en la superioridad masculina.

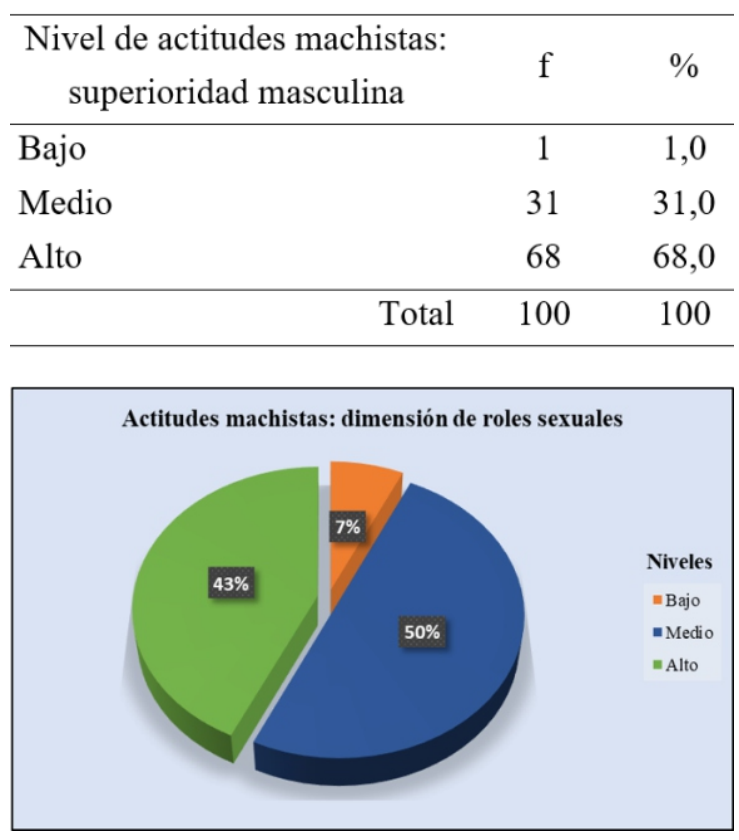

Figura 1. Nivel de actitudes machistas en la dimensión de roles sexuales.

Tabla 4: Comparativo entre las actitudes machistas, asociadas al género, dimensión: dominio masculino

\section{RESULTADOS}




\begin{tabular}{|c|c|c|c|c|}
\hline \multirow{3}{*}{$\begin{array}{l}\text { Actitudes machistas: } \\
\text { Dominio masculino }\end{array}$} & \multicolumn{4}{|c|}{ Género } \\
\hline & \multicolumn{2}{|c|}{ Femenino } & \multicolumn{2}{|c|}{ Masculino } \\
\hline & $\mathrm{f}$ & $\%$ & $\mathrm{f}$ & $\%$ \\
\hline Bajo & 1 & 1,0 & 0 & 0,0 \\
\hline Medio & 17 & 17,0 & 14 & 14,0 \\
\hline Alto & 32 & 32,0 & 36 & 36,0 \\
\hline Tota & 50 & $50 \%$ & 50 & $50 \%$ \\
\hline
\end{tabular}

Tabla 5: Comparativo entre las actitudes machistas, asociadas al género, dimensión: roles domésticos y sociales

\begin{tabular}{|c|c|c|c|c|}
\hline \multirow{3}{*}{$\begin{array}{l}\text { Actitudes machistas: Ro- } \\
\text { les domésticos y sociales }\end{array}$} & \multicolumn{4}{|c|}{ Género } \\
\hline & \multicolumn{2}{|c|}{ Femenino } & \multicolumn{2}{|c|}{ Masculino } \\
\hline & $\mathrm{f}$ & $\%$ & $\mathrm{f}$ & $\%$ \\
\hline Bajo & 4 & 4,0 & 1 & 1,0 \\
\hline Medio & 23 & 23,0 & 21 & 21,0 \\
\hline Alto & 23 & 23,0 & 28 & 28,0 \\
\hline Total & 50 & $50 \%$ & 50 & $50 \%$ \\
\hline
\end{tabular}

Tabla 6. Comparativo entre actitudes machistas asociadas al género, dimensión: roles sexuales

\begin{tabular}{|c|c|c|c|c|}
\hline \multirow{3}{*}{$\begin{array}{l}\text { Actitudes machistas: } \\
\text { Roles sexuales }\end{array}$} & \multicolumn{4}{|c|}{ Género } \\
\hline & \multicolumn{2}{|c|}{ Femenino } & \multicolumn{2}{|c|}{ Masculino } \\
\hline & $\mathrm{f}$ & $\%$ & $\mathrm{f}$ & $\%$ \\
\hline Bajo & 3 & 4,0 & 4 & 4,0 \\
\hline Medio & 30 & 30,0 & 20 & 20,0 \\
\hline \multirow[t]{2}{*}{ Alto } & 17 & 17,0 & 26 & 26,0 \\
\hline & 50 & $50 \%$ & 50 & $50 \%$ \\
\hline
\end{tabular}

Tabla 7. Opinión en la dimensión dominio masculino de la familia awajún.

\begin{tabular}{|l|c|c|c|c|}
\hline \multicolumn{1}{|c|}{$\begin{array}{c}\text { Dimensión dominio } \\
\text { masculino }\end{array}$} & Desacuerdo & Indeciso & $\begin{array}{c}\text { De } \\
\text { acuerdo }\end{array}$ & Total \\
\cline { 2 - 5 } & $\%$ & $\%$ & $\%$ & $\%$ \\
\hline E1 hombre da las órdenes & 16.0 & 3.0 & 81.0 & 100.0 \\
\hline $\begin{array}{l}\text { Fin del matrimonio awajún: } \\
\text { protección de la mujer }\end{array}$ & 23.0 & 4.0 & 73.0 & 100.0 \\
\hline $\begin{array}{l}\text { La mujer debe ser indepen- } \\
\text { diente }\end{array}$ & 34.0 & 10.0 & 56.0 & 100.0 \\
\hline $\begin{array}{l}\text { La mujer awajün no debe } \\
\text { ser autoridad }\end{array}$ & 41.0 & 7.0 & 52.0 & 100.0 \\
\hline $\begin{array}{l}\text { E1 hombre es quien impone } \\
\text { autoridad }\end{array}$ & 19.0 & 6.0 & 75.0 & 100.0 \\
\hline $\begin{array}{l}\text { Los hermanos deben vigilar } \\
\text { a las hermanas }\end{array}$ & 15.0 & 4.0 & 81.0 & 100.0 \\
\hline $\begin{array}{l}\text { Ocupación de la mujer: su } \\
\text { casa y sus hijos }\end{array}$ & 28.0 & 5.0 & 67.0 & 100.0 \\
\hline $\begin{array}{l}\text { La mujer cumple las órde- } \\
\text { nes del esposo }\end{array}$ & 14.0 & 3.0 & 83.0 & 100.0 \\
\hline $\begin{array}{l}\text { La mujer: prohibida ir sola a } \\
\text { fiestas }\end{array}$ & 44.0 & 12.0 & 44.0 & 100.0 \\
\hline $\begin{array}{l}\text { E1 marido debe prohibir que } \\
\text { ella estudie }\end{array}$ & 43.0 & 11.0 & 46.0 & 100.0 \\
\hline
\end{tabular}

Tabla 8. Opinión en la dimensión superioridad masculina, en la familia awajún.

\begin{tabular}{|c|c|c|c|c|}
\hline \multirow{2}{*}{$\begin{array}{c}\text { Dimensión superioridad } \\
\text { masculina }\end{array}$} & Desacuerdo & Indeciso & $\begin{array}{c}\text { De } \\
\text { acuerdo }\end{array}$ & Total \\
\hline & $\%$ & $\%$ & $\%$ & $\%$ \\
\hline $\begin{array}{l}\text { En una reunión social, ella } \\
\text { sólo sirve el masato }\end{array}$ & 45.0 & 4.0 & 51.0 & 100.0 \\
\hline $\begin{array}{l}\text { La mujer debe caminar } \\
\text { siempre detrás del marido }\end{array}$ & 30.0 & 5.0 & 65.0 & 100.0 \\
\hline $\begin{array}{l}\text { Las hijas deben atender a } \\
\text { los hijos }\end{array}$ & 27.0 & 7.0 & 66.0 & 100.0 \\
\hline $\begin{array}{l}\text { No es necesario que las } \\
\text { hijas se eduquen }\end{array}$ & 49.0 & 10.0 & 41.0 & 100.0 \\
\hline $\begin{array}{l}\text { Crees que a futuro las muje- } \\
\text { res deben ser APU }\end{array}$ & 24.0 & 0 & 68.0 & 100.0 \\
\hline $\begin{array}{l}\text { En la casa, seguin él, todo } \\
\text { debe estar en orden }\end{array}$ & 10.0 & 7.0 & 83.0 & 100.0 \\
\hline $\begin{array}{l}\text { La mujer está prohibida } \\
\text { hablar con otra persona }\end{array}$ & 44.0 & 15.0 & 41.0 & 100.0 \\
\hline $\begin{array}{l}\begin{array}{l}\text { El es siempre el jefe de } \\
\text { hogar }\end{array} \\
\end{array}$ & 14.0 & 7.0 & 79.0 & 100.0 \\
\hline El solo debe mitayar y pescar & 33.0 & 8.0 & 59.0 & 100.0 \\
\hline Sölo ella cultiva la chacra & 43.0 & 6.0 & 51.0 & 100.0 \\
\hline
\end{tabular}

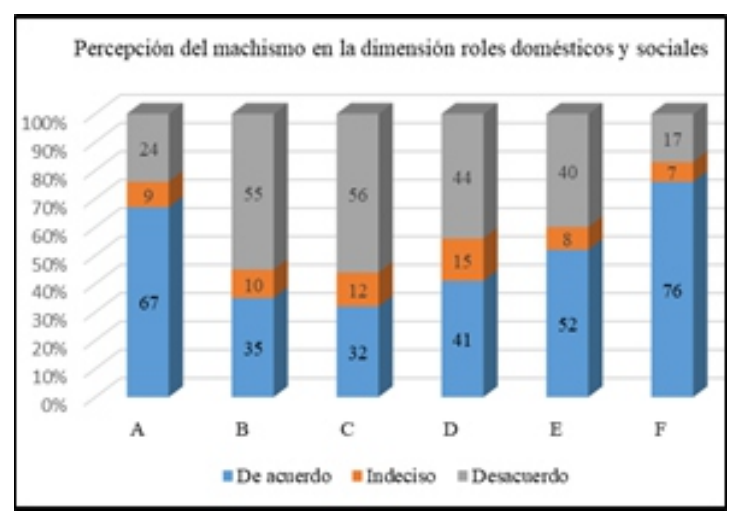

Figura 4: Distribución porcentual de la opinión en la dimensión roles domésticos y sociales

\section{Leyenda:}
A: El marido decide cuantos hijos tener
B: Sólo él debe tener varias mujeres
C: El marido elige la pareja de su hija
D: Sólo ellos deben usar métodos anticonceptivos
E: La mujer debe llegar virgen al matrimonio
F: Él deber de las hijas es servir a sus padres

\section{DISCUSIÓN}

Referirse al tema del "machismo", constituye toda una complejidad, que está asociada a la masculinidad, al acceso a cargos públicos únicamente por los varones, al uso del poder social, económico, entre otros aspectos, para ejercer la dominancia hombre a mujer; que, para Castañeda 
(2007), constituye una forma de violencia familiar. En el caso particular de las actitudes machistas relacionadas al dominio masculino en las familias awajún de Numpatkaim, un elevado $68 \%$ muestra un nivel alto de actitudes machistas en el entorno familiar (Tabla 1). Resultado similar con el estudio de Pintado \& Meza (2015), quienes en su tesis sobre la mujer aguaruna y participación pública; concluye que los varones, según Etsa, les corresponde realizar actividades vinculantes al espacio público y político, básicamente en lo que ellos denominan "el arte para la guerra"; y por esa misma razón, son ellos los que tienen el dominio absoluto ante la mujer, quien cumple un rol de obediencia y sumisión ante el padre de sus hijos.

En la dimensión de roles domésticos y sociales, un $51 \%$ de las familias muestran un alto nivel de machismo en sus actitudes (Tabla 2), lo que se refleja en la práctica, dado que es el hombre el que tiene acceso a los cargos públicos que están contemplados en sus reglamentos, las mujeres no pueden ocupar algún cargo en la comunidad, tampoco acceder al mercado laboral; resultado que muestra similitudes con la investigación de Montserrat y Casas (2017), al referirse al impacto de la violencia machista; quienes concluyen que existe un índice bajo de ocupación laboral entre las mujeres que viven violencia machista; situación que ocasiona que sus hijos se encuentren en un estado de mucha más vulnerabilidad en cuanto al acceso a las necesidades básicas.

En la dimensión de superioridad masculina, se aprecia que el $53 \%$ muestra un alto nivel de actitudes machistas en la familia (Tabla 3); y esa superioridad del hombre sobre la mujer, también son aprendidas por los hijos varones, quienes desde temprana edad, muestran actitudes de predominio sobre sus hijas mujeres; resultado que muestra similitudes en el estudio de Marchal, Brando, Hidalgo y Tomás (2018), relacionado a un instrumento para medir actitudes machistas, violencia y estereotipos en adolescentes; quienes concluyen que los niños presentan mayores creencias legitimadoras de la violencia, que las niñas, y que están atribuidas a factores socioculturales relacionadas a la socialización de género.

Finalmente, al referirse al estudio del machismo en la dimensión de los roles sexuales, en las familias de la comunidad de Numpatkaim, se aprecia que el $43 \%$ muestra un alto índice de actitudes machistas en la dimensión precitada (figura 1). Siguiendo con la descripción de los resultados, se percibe que las actitudes machistas tienen mayor incidencia en el dominio masculino, los varones, ya que el 36\% está en el nivel alto, frente al 32\%, según la opinión de las mujeres (Tabla 4). Asimismo, el 28\% de los varones están en el nivel alto en las actitudes machistas en la dimensión de roles domésticos y sociales, frente al $23 \%$, de las mujeres, en ese mismo nivel (Tabla 5). Haciendo el comparativo entre los roles sexuales, $26 \%$ de varones están en el nivel alto en actitudes machistas, frente al 17\% de mujeres (Tabla 6). Igualmente, en las tablas 7 y 8 ; así como también en la figura 2, se aprecia que en las cuatro dimensiones estudiadas, se impone el predominio de actitudes machistas en los varones; dado que es el varón el que toma las decisiones que tienen que ver con las relaciones de pareja, inclusive para dar el visto bueno al uso de métodos anticonceptivos, y las decisiones para contraer matrimonio o solamente de convivencia; incluso llegado al extremo de que es el varón el que puede tener más de una mujer y la mujer solo debe tener un hombre como pareja; entre otras conductas, que se asocia a los roles sexuales, percibiendo que existen similitudes con el trabajo de investigación de Pintado \& Meza (2015), sobre la mujer aguaruna y su participación pública: una aproximación desde las creencias mitológicas y su influencia en la formación de roles e identidades, en las comunidades aguarunas, Nieva - Amazonas; quienes concluyen que la influencia en los roles, da sentido a sus identidades como mujeres y varones, en patrones diversos de comportamiento y responsabilidades.

\section{CONCLUSIONES}

Los resultados relacionados a identificar las actitudes machistas en las familias de la comunidad de Numpatkaim, Imaza, nos permiten evidenciar que, en la dimensión de dominio masculino, el $68 \%$ de las familias muestran un alto nivel de actitudes machistas en su entorno familiar, el $31 \%$, muestra un nivel medio de machismo y solamente el $1 \%$ un nivel bajo de actitudes machistas. En la dimensión de roles domésticos y sociales, donde, el 51\% de las familias muestran un alto nivel de actitudes machistas en el funcionamiento familiar; el 44\%, un nivel medio; $y$, un escaso $5 \%$, está en un nivel bajo de actitudes machistas.

Los hallazgos en la dimensión de superioridad masculina, el 53\% de las familias muestran un alto nivel de actitudes machistas en la familia; el 44\%, un nivel medio; $y$, un escaso 3\%, está en un nivel bajo.

Finalmente, en la dimensión de roles sexuales, el 43\% muestran un alto nivel de actitudes machistas; el 50\%, está en un nivel medio; $\mathrm{y}$, un escaso $7 \%$, en un nivel bajo. 


\section{REFERENCIAS BIBLIOGRÁFICAS}

Adler, A. (1971). La psicología del machismo. Madrid, España: Press.

Bustamante, M. (1999). Escala de actitudes hacia el machismo (tesis de pregrado). Universidad Nacional Mayor de San Marcos. Lima, Perú.

Castañeda, M. (2007). El machismo invisible regresa. Ciudad de México: Taurus.

Castilla, O. (2015). Los infiernos de la mujer awajún. Mongabay Latam. Recuperado de https://ojo-publico.com/sites/apps/losinfiernos-de-la-mujer-awajun/

De La Rubia y Ramos (2016). Machismo, victimización y perpetración en mujeres y hombres mexicanos. Época III. 23(43). pp. 37-66.

Fuller, N. (2013). Reflexiones sobre el machismo en el Perú. Santiago de Chile: Andrés Bello.

Kohan (1970). Un estudio experimental sobre el machismo. Revista latinoamericana de Psicología, 2(4), pp. 33-66.

Lugo, C. (1985). Violencia y machismo. Nueva Sociedad. (48), pp.40-47.

Marchal, A.M., Brando, C., Hidalgo, M. y Tomás, J. (2018). Diseño y validación de un instrumento para medir actitudes machistas, violencia y estereotipos en adolescentes, (tesis de posgrado). Escuela Universitaria Gimbernat, Sant Cugat del Valles, Barcelona, España.

Ministerio de la mujer y poblaciones vulnerables 2019). Programa nacional contra la violencia familiar y sexual. Lima, Perú: Imprenta del Ministerio de la mujer. $\mathrm{R}$ e c u p e r a d o d e https://www.mimp.gob.pe/contigo/contenid os/pncontigo-articulos.php? codigo $=39$

Montserrat, C. y Casas, F. (2017). El impacto de la violencia machista y procesos de recuperación en la infancia y la adolescencia, en las madres y en las relaciones maternofiliales. Investigación dirigida por el ayuntamiento de Barcelona y la Universidad de Girona, España.

Organización Mundial de la Salud (1998). Violencia contra la mujer. Un tema de salud Prioritario. Recuperado d e https://www.who.int/gender/violence/violen

$$
\text { cia_infopack1.pdf }
$$

Organización de las Naciones Unidas (1995).

Declaración de Beijing: IV Conferencia Mundial sobre las mujeres. Recuperado de https://www.pj.gob.pe/wps/wcm/connect/

Pintado, I. \& Meza, F. (2015). Mujer aguaruna y participación pública: una aproximación desde las creencias mitológicas y su influencia en la formación de roles e identidades, en las comunidades aguarunas, Nieva - Amazonas. (tesis de pregrado). Universidad Nacional del Centro del Perú.

Querol, R. (4 de mayo de 2014). No pregunten a Darwin: tres teorías sobre el origen del machismo. El País. Madrid, España, pp. 1617.

Seymour-Smith, Charlotte (1991). Women have no affines and men no kin: The politics of the jivaroan gender relation. Man. 26(4), pp. 629639.

Torres, L. y Torres, L. M. (1998). Introducción a las ciencias sociales: sociedad y cultura contemporáneas. Ciudad de México: International Thomson Editores, pp. 16-27.

Ubillus, S. (2014). Teoría de las actitudes. Bogotá, Colombia: Nova.

Valderrama, S. (2010). Pasos para elaborar proyectos y tesis de investigación cientifica. Lima, Perú: San Marcos. 